\title{
BIOTECNOLOGÍA
}

\section{Aislamiento, cultivo, viabilidad y evaluación de un consorcio cianobacteria-microalga como acondicionador de suelos}

\author{
Ever Morales Avendaño ${ }^{1}$, Raquel Martínez Pérez ${ }^{2}$ y Gianina Suárez Rodríguez ${ }^{3}$ \\ ${ }^{1}$ Proyecto Becas PROMETEO-SENESCYT \\ ${ }^{2}$ Escuela de Bioanálisis, Pontificia Universidad Católica del Ecuador, Quito, Ecuador \\ ${ }^{3}$ Departamento de Ciencias de la Vida y Agricultura. Universidad de las Fuerzas Armadas-ESPE \\ evermster@gmail.com_Ｒecibido: 2014-06-30; aceptado: 2014-10-30
}

\section{RESUMEN.-}

Se aislaron microalgas y cianobacterias de rizósfera de leguminosas, cultivadas en la Parroquia Poaló, Latacunga, Provincia de Cotopaxi-Ecuador. A partir de las cuales, se obtuvo un consorcio conformado por Anabaena sp., Nostoc sp. y Chlamydomonas sp. (3:1:3) y estabilizados en cultivos discontinuos alimentados. El contenido de materia orgánica (MO), N, P y Sen el suelo previo al bioensayo fue de $1.93 \%, 0.10 \%, 49.6$ y 13.08 ppm; respectivamente. Los tratamientos para la evaluación del consorcio como acondicionador de suelos, correspondieron a un control (TE), fertilizante químico (TFQ), consorcio (TCC) y fertilizante químico + consorcio (TCF). Los resultados evidenciaron que el tratamiento (TCF) brindó el contenido más alto de $\mathrm{MO}, \mathrm{N}$ y S con $4.5 \%, 0.23 \%$ y 109.8 ppm (>0.05); respectivamente; seguido del suelo inoculado con el consorcio, con $2.2 \%, 0.11 \%$ y 90.2 ppm de MO, N y P; respectivamente. En el suelo con solo el consorcio (TCC) se registró el mayor crecimiento espontáneo de plantas silvestres respecto al resto de los tratamientos $(>0.05)$. Se establece un protocolo para la producción de consorcios con cianobacterias fijadoras de nitrógeno en condiciones de laboratorio. Su aplicación en biotecnología agrícola permite ampliar el espectro de microorganismos utilizados como acondicionadores de suelos.

PALABRAS CLAVES: acondicionador, biotecnología agrícola, consorcio cianobacteria-microalga, cultivo, química suelo

\begin{abstract}
.-
Microalgae and cyanobacteria from legume rhizosphere were isolated, cultured in Poalo, Latacunga, Cotopaxi Province, Ecuador. From which, a consortium of Anabaena sp. Nostoc sp. and Chlamydomonas sp. (3:1:3) was obtained, and stabilized in fed batch cultures. The content of organic matter (OM), $\mathrm{N}, \mathrm{P}$ and $\mathrm{S}$ in the soil prior to the bioassay was $1.93 \%, 0.10 \%, 49.6$ and $13.08 \mathrm{ppm}$; respectively. Treatments for the evaluation of the consortium as a soil conditioner, corresponded to a control (TE), chemical fertilizer (TFQ) Consortium (TCC) and chemical fertilizer + consortium (TCF). The results showed that treatment (TCF) gave the highest content of $\mathrm{OM}, \mathrm{N}$ and $\mathrm{S}$ with $4.5 \%, 0.23 \%$ and $109.8 \mathrm{ppm}$ (>0.05); respectively; followed by soil inoculated with the consortium, with $2.2 \%$,
\end{abstract}


$0.11 \%$ and $90.2 \mathrm{ppm} \mathrm{OM}, \mathrm{N}$ and P; respectively. On the ground with only the consortium (TCC) increased spontaneous growth of wild plants from the rest of the treatments $(>0.05)$ was recorded. A protocol for the production of consortia with nitrogen-fixing cyanobacteria in laboratory conditions is established. Its application in agricultural biotechnology broadens the range of organisms used as soil conditioners.

KEYWORDS: agricultural biotechnology, conditioner, chemistry soil, cyanobacteria-microalgae consortium

\section{INTRODUCCIÓN}

El uso indebido de los agroquímicos, ocasiona impactos negativos en suelos agrícolas, con el consiguiente desgate físico, pérdida de la base nutrimental, húmica y de la actividad microbiana. Asimismo, ha propiciado bajas sensibles de la productividad de la mayoría de los cultivos, severos desbalances en los agroecosistemas y contaminación ambiental, provocando impactos negativos en la salud de los agricultores y consumidores finales (Suquilanda, 2008).

Los fertilizantes convencionales presentan baja eficiencia $(\leq 50 \%)$ para ser asimilados por los cultivos y además, el excedente no incorporado por las plantas trae un impacto ambiental adverso; tal como contaminación de mantos acuíferos con nitratos, eutrofización, lluvia ácida y calentamiento global (Armenta et al., 2010).

Los biofertilizantes representan una importante alternativa para la sustitución de fertilizantes químicos; ya que proporcionan mayor rentabilidad económica, aumentan la productividad de los cultivos, reducen los efectos de la fertilización química sobre el medio ambiente y la salud (Red BIOFAG, 2007). Es por ello, que el uso eficaz de biofertilizantes microbianos en agricultura, se propone como una de las tecnologías alternativas de producción menos contaminantes y ecológicamente más racionales, como es el uso de los recursos microbiológicos del suelo en la agricultura (Aguirre et al., 2009). En tal sentido, presentan una expectativa, especialmente para lograr una menor dependencia de los fertilizantes químicos y una mayor disponibilidad de nutrientes (Benitez, 2005).
Entre los beneficios del uso de microorganismos en la agricultura están su capacidad de fijación de nitrógeno atmosférico de una forma simbiótica o asimbiótica, descomponer residuos orgánicos, desintoxicar plaguicidas, supresión de enfermedades en las plantas, aportar nutrientes al suelo y producir compuestos bioactivos como vitaminas y hormonas que estimulan el crecimiento de las plantas (Terry y Leiva, 2005). De tal manera que, pueden ser identificados microorganismos fotosintéticos, entre la microbiota del suelo y lograr su crecimiento y cultivo en presencia de humedad, nutrientes básicos e iluminación natural con la finalidad de seleccionar consorcios con cianobacterias fijadoras asimbióticas de nitrógeno con potencial para ser utilizados como acondicionadores de suelos.

En muchos países ya se han realizado estudios con estos microorganismos e incluso se está comenzando a producirlos a escala industrial, de ahí la importancia de evaluar el efecto biofertilizante de las cianobacterias y microalgas en nuestro medio (Monteros e Iglesias, 2005). En el presente estudio se valora el aporte de materia orgánica y de nutrientes de cianobacterias fijadoras de nitrógeno y de microalgas en consorcios aislados previamente de suelos cultivados con leguminosas para ser evaluados como fuente de nutrientes en estos suelos agrícolas.

\section{MATERIALES Y MÉTODOS}

\section{Toma de muestras.-}

Se recolectaron varias plantas leguminosas de fréjol (Phaseolus vulgaris), haba (Vicia faba), chocho 
(Lupinus mutabilis) y de arveja (Pisum sativum) con su raíz y rizósfera. Estas muestras fueron colectadas de suelos areno arcillosos, en la Parroquia Poaló (2.918 m.s.n.m.; y entre $00^{\circ} 08^{\prime} 21^{\prime \prime} \mathrm{W}$ y $00^{\circ} 54^{\prime} 45^{\prime \prime}$ S), ubicada al Noroeste de la ciudad de Latacunga, Provincia de Cotopaxi, Ecuador. Posteriormente estas plantas fueron transportadas en bolsas de plástico con cierre hermético al laboratorio y refrigeradas a $4^{\circ} \mathrm{C}$ para la toma de las muestras respectivas.

Incubación de muestras en medio de cultivo sólido.-

Para el crecimiento y aislamiento de cianobacterias de la rizósfera de leguminosas se utilizaron dos medios de cultivo. Fertilizante foliar Nitrofoska ( $\mathrm{N}$ total 10\%, $\mathrm{P}_{2} \mathrm{O}_{5} 4.0 \%, \mathrm{~K}_{2} \mathrm{O} 7.0 \%, \mathrm{MgO}$ $2.0 \%$, S 0.8\%, Fe 70ppm, B 11ppm, Cu 12ppm, Mn 8ppm, Zn 2 ppm, Co 12ppm, Mo 1ppm) y BG-11 ${ }_{0}$ (sin nitrógeno: $\mathrm{K}_{2} \mathrm{HPO}_{4} \cdot 3 \mathrm{H}_{2} \mathrm{O} 40 \mathrm{gl}^{-1}$, Mg$\mathrm{SO}_{4} .7 \mathrm{H}_{2} \mathrm{O} 75 \mathrm{gl}^{-1}, \mathrm{CaCl}_{2} \cdot 2 \mathrm{H}_{2} \mathrm{O} 36 \mathrm{gl}^{-1}$, ácido cítrico $6 \mathrm{gl}^{-1}$, citrato de amonio $6 \mathrm{gl}^{-1}$, EDTA $1 \mathrm{gl}^{-1}, \mathrm{Na}$ ${ }_{2} \mathrm{CO}_{3} 20 \mathrm{gl}^{-1}, \mathrm{H}_{3} \mathrm{BO}_{3} 0.29 \mathrm{gl}^{-1}, \mathrm{MnCl}_{2} \cdot 4 \mathrm{H}_{2} \mathrm{O} 0.18 \mathrm{gl}^{-1}$, $\mathrm{ZnSO}_{4} .7 \mathrm{H}_{2} \mathrm{O} 0.02 \mathrm{gl}^{-1}, \mathrm{Na}_{2} \mathrm{MoO}_{4} .5 \mathrm{H}_{2} \mathrm{O} 0.04 \mathrm{gl}^{-1}$, $\mathrm{CuSO}_{4} \cdot 5 \mathrm{H}_{2} \mathrm{O} 0.08 \mathrm{gl}^{-1}, \mathrm{Co}\left(\mathrm{NO}_{3}\right) \cdot 6 \mathrm{H}_{2} \mathrm{O} 0.005 \mathrm{gl}^{-1}$, $\left.\mathrm{FeCl}_{3} 0.02 \mathrm{gl}^{-1}\right)$, como medio selectivo para cianobacterias (Rippka, et al., 1979).

Para la incubación de las muestras de raíces de leguminosas, se procedió a colocarlas en dos cajas de Petri estériles; en una de las cuales se añadió 5 mililitros $(\mathrm{ml})$ de medio de cultivo Nitrofoska, y en la otra, $5 \mathrm{ml}$ del medio BG11, con un réplica de cada caja. Además, se tomó $5.0 \mathrm{~g}$ del suelo asociado a las raíces de cada una de las plantas leguminosas y se colocó en medio de cultivo Nitrofoska, y a la otra muestra de suelo en $5 \mathrm{~mL}$ del medio BG11 $1_{0}$ en dos cajas de Petri respectivas. Estas fueron incubadas bajo iluminación continua con una lámpara fluorescente en posición lateral marca Philips Dayligth de $40 \mathrm{~W}$.

Las microalgas y cianobacterias crecidas en las muestras y mantenidas durante 30 días fueron aisladas en medio sólido BG- $11_{0^{\prime}}$ usando la técnica de agotamiento bajo condiciones asépticas. Luego de dos semanas de incubación en condiciones de luminosidad con un fotoperíodo 12:12, y a una temperatura de $20^{\circ} \mathrm{C} \pm 2$, se observaron nuevamente al microscopio. Las poblaciones de microalgas y cianobacterias fueron sometidas a resiembra por agotamiento en medio sólido BG-11 0 con Bicarbonato de Sodio para su aislamiento. A partir de claves taxonómicas fueron identificadas las cianobacterias (Komárek y Anagnostidis, 2005), las microalgas Chlorophyta (Eaton et al., 1995) y Bacillariophyceae (Rumrich et al., 2000).

Condiciones de cultivo, determinación del crecimiento y contenido de pigmentos del consorcio con cianobacterias fijadoras de nitrógeno.-

Las cepas seleccionadas de cianobacterias se escalaron con medio BG-11 0 líquido en tubos de ensayo de $10 \mathrm{ml}$. Posteriormente se inició el sistema de cultivo discontinuo en matraces Erlenmeyer de aproximadamente $500 \mathrm{ml}$ de capacidad, con un inóculo de $100 \mathrm{ml}$ de cultivo con cada una de las 6 diferentes cepas de cianobacterias que fueron aisladas.

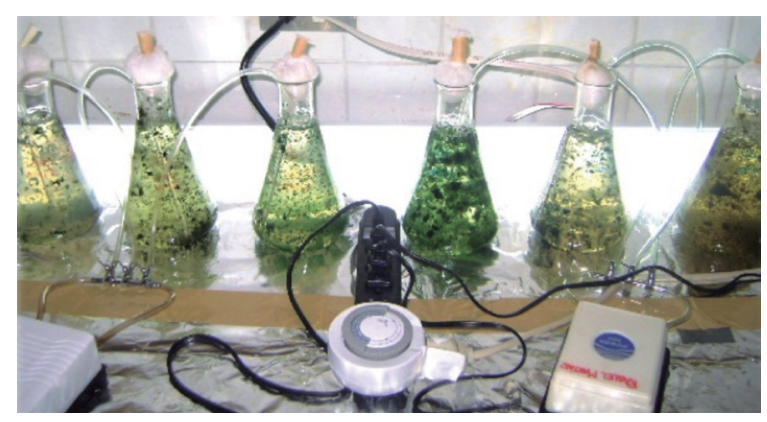

Figura 1. Sistema de cultivo discontinuo de las cianobacterias aisladas.

Luego, estos cultivos fueron escalados hasta 6.01 , mediante un sistema de cultivo discontinuo alimentados semanalmente con 1.01 de medio de cultivo BG- $11_{0^{\prime}}$ y mantenidos con una aireación de $5.0 \mathrm{ml} . \mathrm{s}^{-1}$, a $20^{\circ} \mathrm{C} \pm 2$, a una intensidad luminosa de $156 \mu$ molq. ml.s $\mathrm{s}^{-1} \mathrm{~s}^{-2}$, fotoperiodo de 12:12 h y a $\mathrm{pH} 8$ (Fig.2).

Finalmente, se procedió a realizar un agrupamiento de las 6 cepas de cianobacterias, tomando 1.01 de cada cepa y 2.01 de medio de cultivo BG11 $1_{0}$ y se continuó manteniendo el sistema de cultivo discontinuo alimentado durante 30 días 
bajo los mismos parámetros de luminosidad, de 45.01 con la adición 2.01 del medio de cultivo aireación y $\mathrm{pH}$ hasta alcanzar un volumen final cada 5 días.

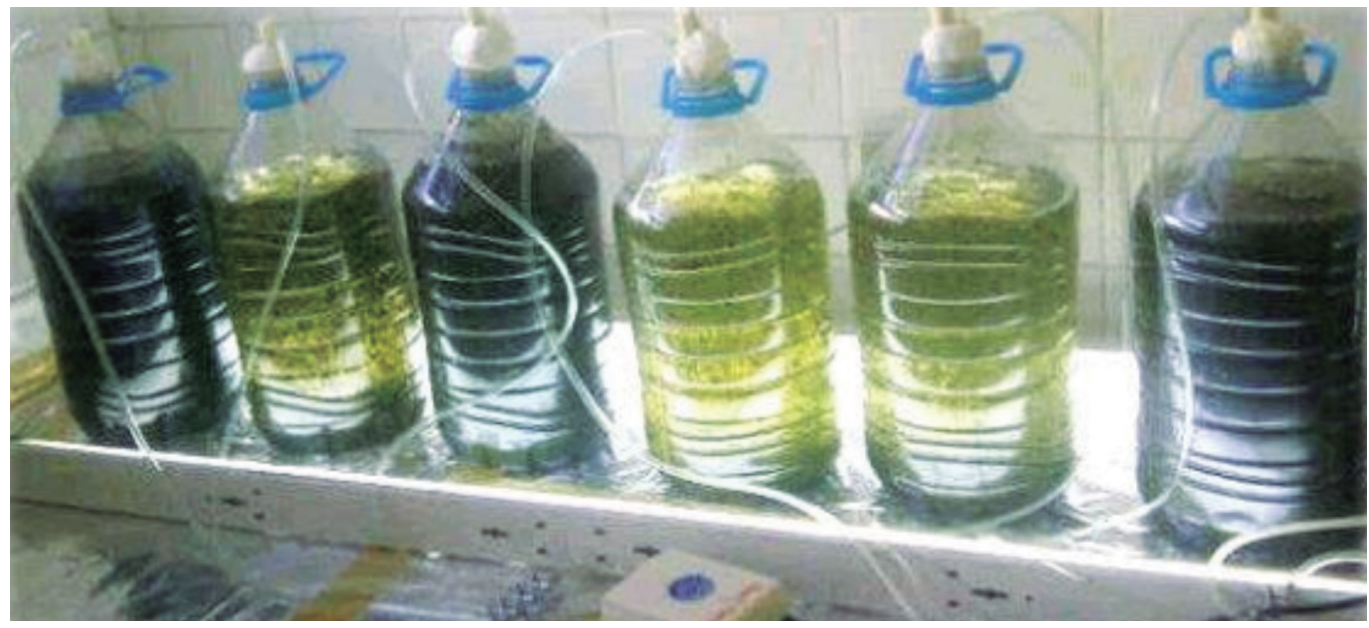

Figura 2. Escalamiento de cultivos discontinuos de cianobacterias a 6.0.1.

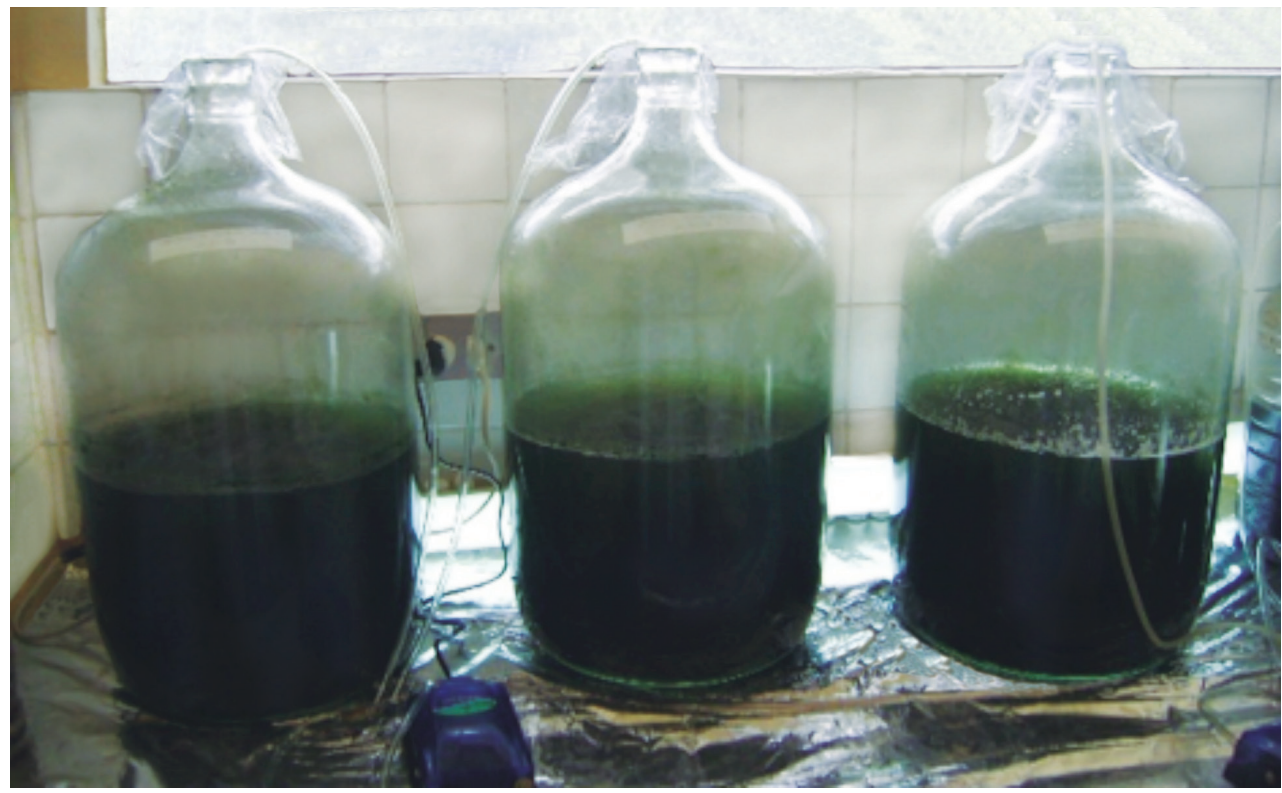

Figura 3. Consorcios de cianobacterias en cultivos discontinuos alimentados con BG110.

El crecimiento del consorcio se siguió a partir de $5.0 \mathrm{ml}$ por triplicado cada 5 días y mediante turbidez a $750 \mathrm{~nm}$ en un spectronic (20D Milton Roy, Bausch \& Lomb, USA, New York). El contenido de pigmentos liposolubles fue determinado por triplicado a un volumen de 2.0 $\mathrm{ml}$ y mediante el método Marker et al. (1980) y de Strickland y Parsons (1972), para clorofila a y carotenoides respectivamente. Mientras que, la concentración de ficocianina, aloficocianina y ficoeritrina se determinó de acuerdo a las fórmulas propuestas por Bennett y Bogorad (1973).

\section{Cosecha del consorcio de cianobacteria-micro- alga (TCC).-}

Se procedió a cosechar el consorcio, mediante 
sedimentación natural de la biomasa producida en cultivos discontinuos alimentados. Posteriormente, se decantó el sobrenadante líquido por varias ocasiones hasta obtener únicamente la biomasa a un volumen de 1.125l. Luego, $250 \mathrm{ml}$ de este volumen, se distribuyó en tres frascos de vidrio y se aforó a $400 \mathrm{ml}$ con agua destilada; lo cual equivale a una dilución del $40 \%$ (v/v) (Fig. 4). Este volumen representa el inóculo que fue distribuido en la muestra de suelo de $2,0 \mathrm{Kg}$, para quedar completamente humedecido.

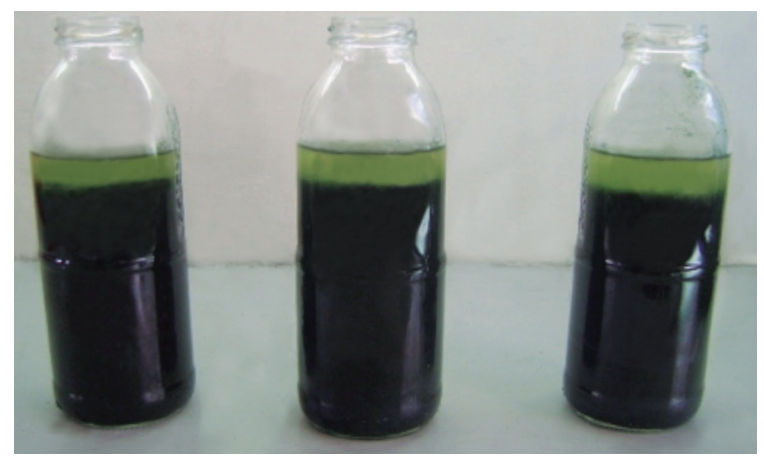

Figura 4. Consorcio de cianobacterias para ser inoculado en suelos.

Preparación del tratamiento con el fertilizante químico (TFQ).-

El fertilizante comercial químico utilizado para el ensayo fue el 10-20-10, cuyos números indican el porcentaje de nitrógeno, $\mathrm{P}_{2} \mathrm{O}_{5}$ y $\mathrm{K}_{2} \mathrm{O}$; respectivamente en 100 libras del fertilizante (Masabni y Lillard, 2011). Para su preparación se pesó 4,0g del fertilizante químico y se colocó en botellas de vidrio con $400 \mathrm{ml}$ de agua destilada; lo cual corresponde a una concentración del fertilizante del $1 \%(\mathrm{p} / \mathrm{v})$.

\section{Preparación del tratamiento con consorcio + el fertilizante químico (TCF).-}

Se mezcló el consorcio a un volumen de $150 \mathrm{ml}$ y 2,0g de fertilizante químico. Posteriormente se ajustó el volumen final a $400 \mathrm{ml}$ con agua destilada, equivalente a una concentración del fertilizante de $0,5 \%$ (p/v) y a una dilución equivalente para el volumen del consorcio del $80 \%$ (v/v).

\section{Aplicación de tratamientos en el suelo.-}

Los tratamientos que se consideraron para el pre- sente estudio fueron los siguientes: testigo: suelo sólo con agua destilada (TE); suelo inoculado con el consorcio cianobacteria-microalga (TCC); suelo inoculado con el fertilizante comercial químico (TFQ); suelos inoculado con el fertilizante químico + consorcio cianobacteria-microalga (TCF).

Se utilizaron bandejas plásticas de aproximadamente $30 \mathrm{~cm}$ x $30 \mathrm{~cm} \times 20 \mathrm{~cm}$, con $2.0 \mathrm{~kg}$ de suelo y los respectivos tratamientos: TE, TCC, TFQ y TCF fueron iniciados con un volumen de $400 \mathrm{ml}$ y con tres réplicas.

\section{Evaluación de la viabilidad del consorcio ciano- bacteria-microalga en suelos.-}

Posterior a la inoculación del suelo se monitoreó las poblaciones de cianobacterias y microalgas presentes en cada tratamiento y durante cada semana y hasta la finalización del experimento en la quinta semana. Para tal fin, y de acuerdo al uso de una malla cuadriculada colocada sobre la superficie de cada suelo; se tomaron muestras compuestas, de aproximadamente $0,5 \mathrm{~g}$ de cinco diferentes puntos de cada tratamiento (Fig. 5). Las muestras fueron colocadas en tubos de ensayo con 3,0 $\mathrm{ml}$ de agua destilada, para posteriormente ser identificadas al microscopio, las microalgas y cianobacterias presentes en cada tratamiento. Cada dos días fueron hidratados los tratamientos a fin de mantener las condiciones de humedad y favorecer el crecimiento de microorganismos fotosintéticos en dichos suelos.

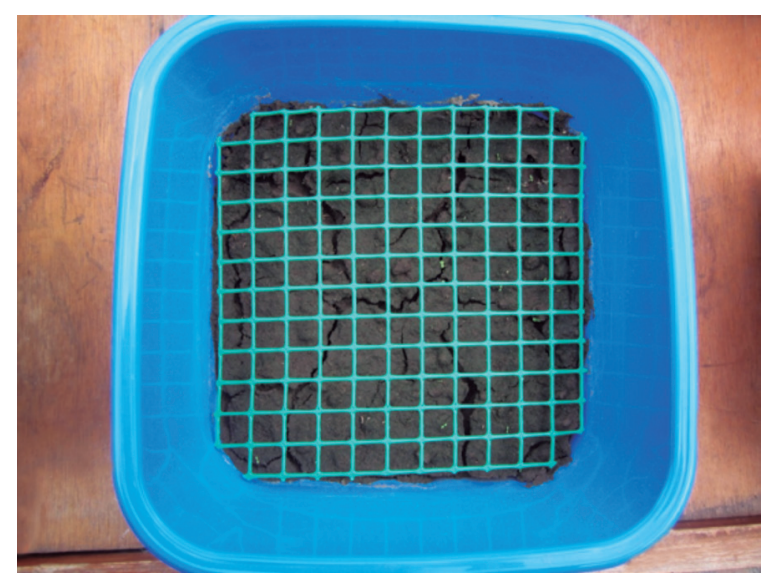

Figura 5. Rejilla para muestreo de microalgas y cianobacterias en suelos con los diferentes tratamientos. 
Los muestreos se realizaron a los 8, 15, 22, 29 y 36 días para el seguimiento de la presencia de los microorganismos fotosintéticos que conformaron el consorcio y de los no pertenecientes a dicho consorcio, observados durante el período de estudio.

La población de taxa de cianobacterias y microalgas fueron expresadas en "cruces" y se les atribuyó valores numéricos según su abundancia (Morales et al., 2013): escasa (una cruz), moderada (desde dos hasta cinco cruces) y abundante (a partir de seis cruces).

Crecimiento de plantas en los tratamientos.Adicionalmente, se realizó un monitoreo del número de plántulas crecidas espontáneamente, sin haber inoculado sus semillas en los diferentes tratamientos. Al día 36 , se procedió a determinar el número de plantas que había en cada uno de los tratamientos y se las identificó a nivel de género con la ayuda de material bibliográfico del Herbario Nacional del Ecuador (QCNE).

\section{Muestreo y análisis químico del suelo.-}

Se tomaron submuestras representativas del suelo cultivado con las plantas leguminosas, haciendo un recorrido en forma de zig-zag (Báscones, 2004) hasta obtener 2,0 kg de suelo. Se identificó la muestra con todos los datos necesarios (provincia, cantón, parroquia, altura, altitud, longitud, manejo agronómico, tipo de leguminosas cultivadas). A la muestra de suelo se le retiró las impurezas como piedras o ramas, luego se secaron en bandejas plásticas bajo iluminación solar y tamizado en mallas número 12 de 1,0 mm (Arango y Pérez, 2005).

Para la fase experimental a nivel de laboratorio se empleó un diseño completamente al azar (DCA) en el cual se investigó la influencia de los diferentes tratamientos inoculados en el suelo, en comparación a una muestra de suelo antes del estudio con los diferentes tratamientos, como control a través de análisis químicos $(\mathrm{B}, \mathrm{Na}, \mathrm{S}$, $\mathrm{Mn}, \mathrm{N}, \mathrm{P}, \mathrm{Mg}, \mathrm{Zn}, \mathrm{Ca}, \mathrm{Cu}$ ), materia orgánica, capacidad de intercambio de cationes y $\mathrm{pH}$. Las muestras fueron enviadas al laboratorio de suelos de Agrocalidad para la determinación de nutrientes (B, Na, S, K, Mn, N, P, Mg, Zn, Fe, Ca, $\mathrm{Cu})$, materia orgánica $(\mathrm{MO})$, capacidad de intercambio de cationes y $\mathrm{pH}$.

\section{Análisis estadístico.-}

Se realizó un análisis de varianza (ANOVA) de un factor. Los resultados se analizaron mediante el empleo del software estadístico SPSS 2.0 y las pruebas de significancia de Tukey al 5\%.

\section{RESULTADOS}

Identificación de microalgas y cianobacterias en suelos y rizósfera de plantas leguminosas.Durante un período de tres meses se monitoreó la presencia de microalgas y cianobacterias, tanto en muestras de suelos como en la rizósfera de leguminosas incubadas con el medios de cultivo BG- $11_{0}$ y el fertilizante foliar Nitrofoska. Se identificaron siete taxa de microalgas y siete de cianobacterias en las muestras de los suelos y de rizósfera de las leguminosas evaluadas. Entre las microalgas predominaron las clorofitas en un $85,71 \%$; y con Navícula sp. como única representante de las diatomeas. Las cianobacterias observadas se caracterizaron por ser todas filamentosas del orden nostocales (Anabaena sp., Calothrix sp. y Nostoc sp.) y oscillatoriales (Leptolyngbya sp., Lyngbya sp., Phormidium sp. y Pseudanabaena sp.) tanto asociadas a rizósfera como en suelos cultivados con plantas leguminosas (Tabla 1). 
Tabla 1. Registro de taxa de microalgas y cianobacterias asociadas a rizósfera de leguminosas.

\begin{tabular}{ll}
\hline & Chlamydomonas sp. \\
& Chlorella sp. \\
Chlorococcum sp. & Desmodesmus sp. \\
Mavicula sp. \\
Pediastrum sp. \\
Scenedesmus sp. \\
\\
Anabaena sp. \\
Calothrix sp. \\
Lyngbya sp. \\
Nostoc sp. \\
Phormidium $\mathrm{sp}$. \\
Pseudanabaena sp. \\
Leptolyngby sp. \\
\hline
\end{tabular}

El uso del medio de cultivo influenció en el número de taxa observadas tanto en suelos como en la rizósfera de las leguminosas. Es decir, en la rizósfera y suelo cultivado con chocho (Lupinus mutabilis) se observaron 6 taxa, tres de microalgas $y$ tres de cianobacterias en presencia del fertilizante Nitrofoska y del medio selectivo BG$11_{0}$; respectivamente. En cuanto a la expresión de taxa de cianobacterias, esta fue observada tanto con Nitrofoska como con BG-11, aun cuando este es un medio selectivo para cianobacterias. La mayor diversidad se detectó en haba (Vicia faba); de las cuales siete correspondieron a cianobacterias y tres de estas con heterocistos (Anabaena, Calothrix y Nostoc). En cambio, en arveja (Pisum sativum) se identificó el menor número de taxa con solo cuatro. De tal manera que, el orden descendente según número de taxa fue el siguiente: haba $>$ chocho $>$ fréjol $>$ arveja. En cambio, al comparar el número de taxa observados entre la rizósfera y suelos cultivados con las respectivas leguminosas, se distinguieron más taxa en la rizósfera en proporción 7:5 frente al suelo cultivado. En cuanto al efecto del tipo de medio de cultivo utilizado, se observó una tendencia a la inducción del crecimiento de cianobacterias y de microalgas, por parte del medio BG-11 y Nitrofoska, respectivamente.

\section{Aislamiento y cultivo del consorcio de ciano- bacterias.-}

De las cianobacterias identificadas del orden nostocales, se aislaron Anabaena sp. ( Fig. 6) y Nostoc sp. (Fig. 7) y caracterizadas por ser las más abundantes y presentes en la mayor parte de las muestras de la rizófera y de suelos incubados con medio BG-11. Ambas cianobacterias fueron sometidas a un proceso de escalamiento hasta obtenerse un consorcio estabilizado y conformado por Anabaena sp., Nostoc sp. y Chlamydomonas sp. en proporción de 3:1:3 respectivamente, en cultivos discontinuos alimentados.

Dicho consorcio cultivado hasta 45.0 1, se caracterizó por presentar una absorbancia de 1.36 (750 nm), un contenido de clorofila $a$ y carotenoides de 1.96 $\mu \mathrm{g} . \mathrm{ml}^{-1}$ y $2.29 \mu \mathrm{g} . \mathrm{ml}^{-1}$; respectivamente. Mientras que, también alcanzó un contenido de ficocianina, aloficocianina y ficoeritrina produjo $1.87 \mu \mathrm{g} \cdot \mathrm{ml}^{-1}$, $1.83 \mu \mathrm{g} . \mathrm{ml}^{-1}$ y $1.76 \mu \mathrm{g} . \mathrm{ml}^{-1}$; respectivamente. 
Tabla 2. Microalgas y cianobacterias asociadas tanto a suelos como en rizósfera de leguminosas de acuerdo al tipo de medio de cultivo.

\begin{tabular}{|c|c|c|c|}
\hline Planta & Muestra & Medio de cultivo & Microalgas/ cianobacterias \\
\hline \multirow{14}{*}{$\begin{array}{l}\text { Chocho } \\
\text { (Lupinus mutabilis) }\end{array}$} & \multirow[t]{9}{*}{ Rizósfera } & \multirow[t]{6}{*}{ Nitrofoska } & Anabaena sp. \\
\hline & & & Chlamydomonas sp. \\
\hline & & & Chlorella sp. \\
\hline & & & Desmodesmus sp. \\
\hline & & & Navícula sp. \\
\hline & & & Scenedesmus sp. \\
\hline & & \multirow{3}{*}{ BG11 } & Chlamydomonas sp. \\
\hline & & & Lyngbya sp. \\
\hline & & & Nostoc sp. \\
\hline & \multirow{5}{*}{ Suelo } & \multirow{3}{*}{ Nitrofoska } & Chlamydomonas sp. \\
\hline & & & \\
\hline & & & Lyngbya sp. \\
\hline & & \multirow[b]{2}{*}{ BG11 } & Anabaena sp. \\
\hline & & & Chlamydomonas sp. \\
\hline \multirow{9}{*}{$\begin{array}{c}\text { Fréjol } \\
\text { (Phaseolus vulgaris) }\end{array}$} & \multirow{5}{*}{ Rizósfera } & \multirow[b]{2}{*}{ Nitrofoska } & Chlamydomonas sp. \\
\hline & & & Pediastrum sp. \\
\hline & & \multirow{3}{*}{ BG11 } & Chlamydomonas sp. \\
\hline & & & Lyngbya sp. \\
\hline & & & Pseudanabaena sp. \\
\hline & \multirow{4}{*}{ Suelo } & \multirow[b]{2}{*}{ Nitrofoska } & Chlamydomonas sp. \\
\hline & & & Chlorella sp. \\
\hline & & \multirow[b]{2}{*}{ BG11 } & Chlamydomonas sp. \\
\hline & & & Lyngbya sp. \\
\hline
\end{tabular}




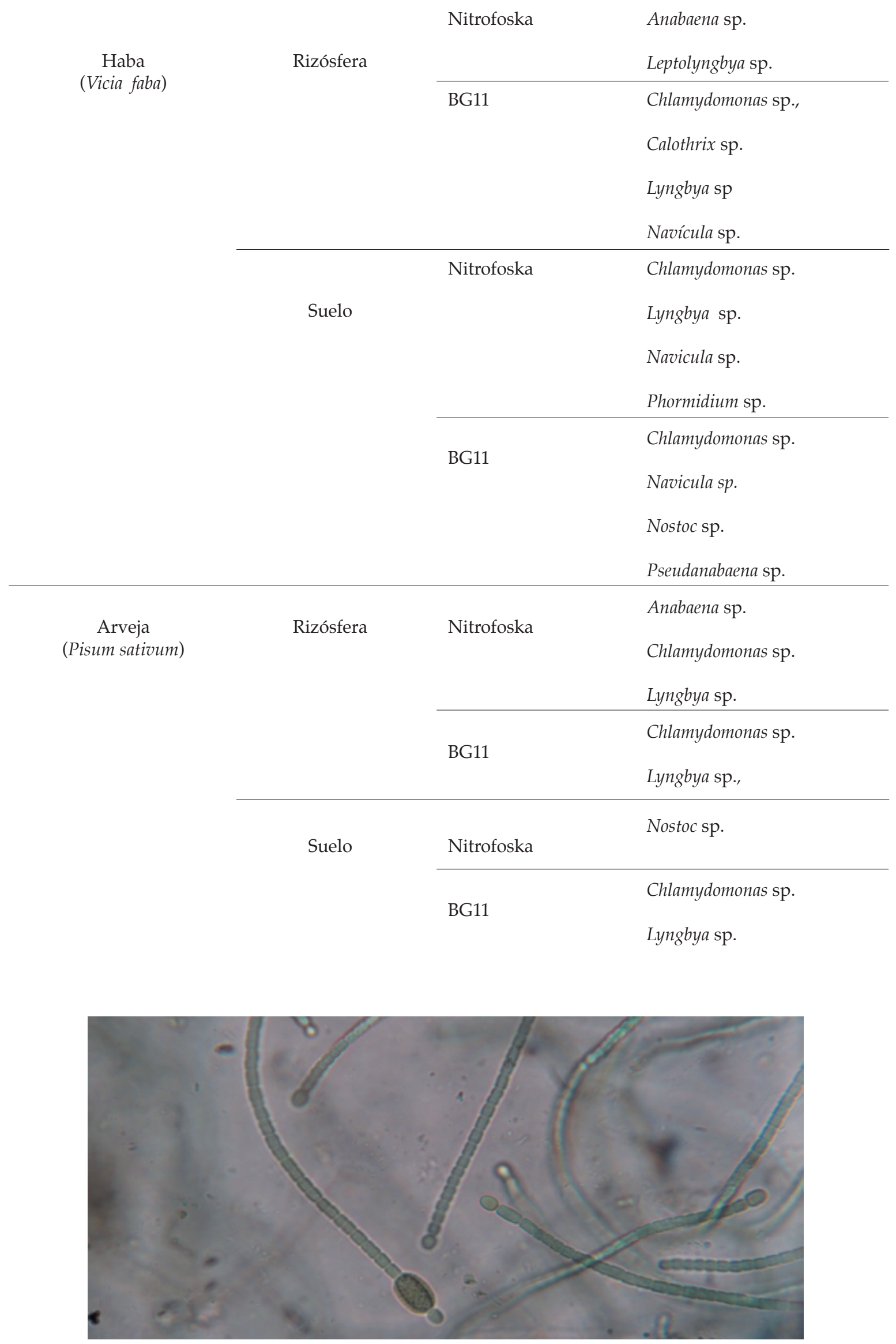

Figura 6. Anabaena sp. y Cylindrospermus sp. con acineto muy evidente, adjunto al heterocisto terminal (40X). 


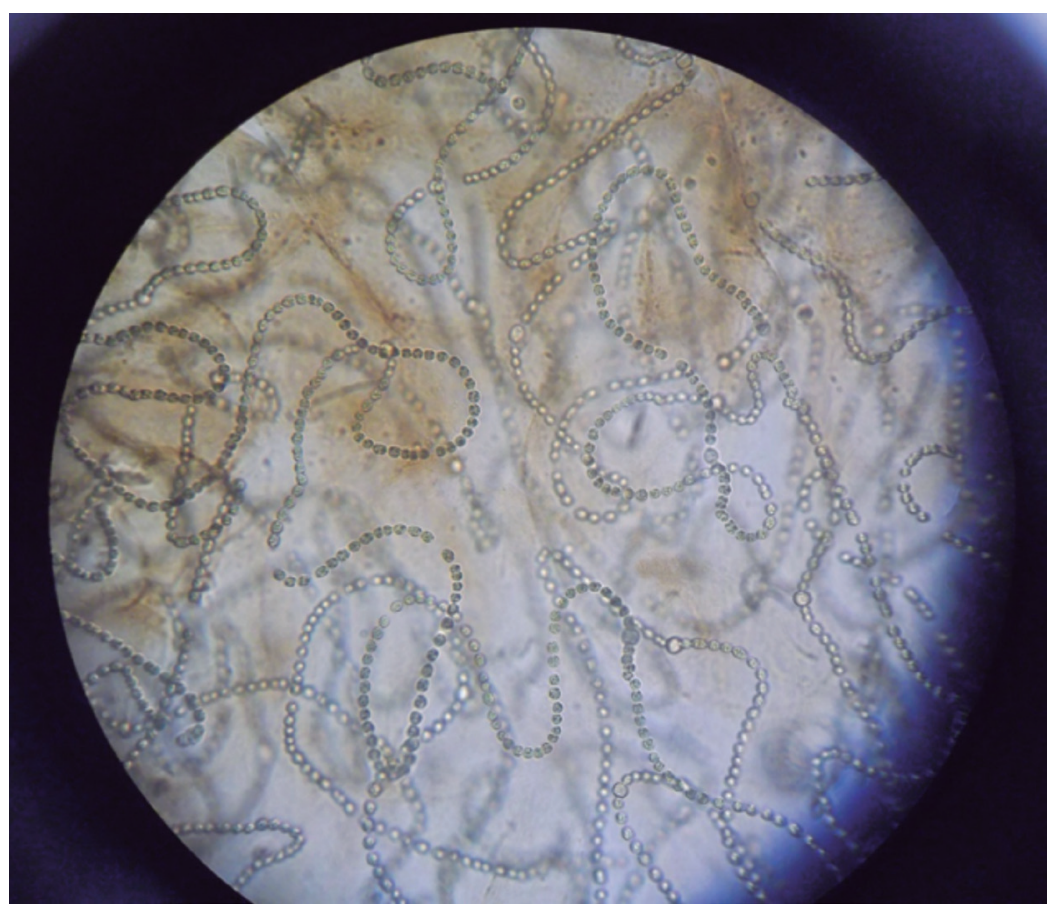

Figura 7. Nostoc sp. (40X)

Análisis de viabilidad del consorcio inoculado en el suelo.-

Los tres microorganismos fotosintéticos integrantes del consorcio e inoculados en el suelo (TCC) se mantuvieron durante el período de estudio (Tabla 3). Se destacó una elevada y continua presencia de Anabaena sp., seguida de la microalga Chlamydomonas sp. y luego de Nostoc sp. La presencia de Chroococcus sp. y de Navícula sp. se observó desde el día 8 hasta el 36. Mientras que, la de Lyngbya sp. y Nitzschia sp., a partir del día 29. Esto significa que, además del consorcio inoculado (3 taxa), se observaron adicionalmente otras cuatro taxa. El mayor índice numérico al final del estudio, se mantuvo con Anabaena sp. (3) y seguido de Chroococcus sp. y Lyngbya sp. con 2 (Tabla 4).

En las muestras de suelos inoculadas con el consorcio y el fertilizante químico (TCF), solo mantuvo la presencia de Anabaena sp. con el mayor índice numérico de 3 , y de Chlamydomonas sp. con el valor de 1. No obstante, Nostoc sp. no reflejó viabilidad aparente hasta el final del estudio. En este tratamiento también fue observado Navícula sp., Lyngbya sp. y Nitzschia sp.; las cuales registraron en el día 36, un crecimiento moderado y con un índice numérico de 2.

En el suelo control, solo se observó crecimiento de Chroococcus sp., Lyngbya sp., Navícula sp., Nitzschia sp. y Chlamydomonas sp.; aunque esta microalga clorofita también conformaba parte del consorcio inoculado. Esto confirma que estas cianobacterias y microalgas, constituyen parte de la biota fotosintética de estos suelos, y con un predominio de Lyngbya sp. y Nitzschia sp., las cuales exhibieron un índice numérico de 2. En cambio, en los suelos inoculados con el fertilizante químico comercial (TCQ), no se observó presencia de cianobacterias y de microalgas durante el período de estudio (Tabla 4).

Presencia de plantas silvestres en los tratamientos.-

En la etapa final del ensayo se evidenció en los diferentes tratamientos, la presencia espontánea de pequeñas plantas. Entre las cuales se destacaron Barnadesia sp., Chromolaena sp., Pennisetum clandestinum, Trifolium repens y Coriandrum sp. En el tratamiento TCC se observaron el mayor número de taxa y de plantas con 7 y 111; 


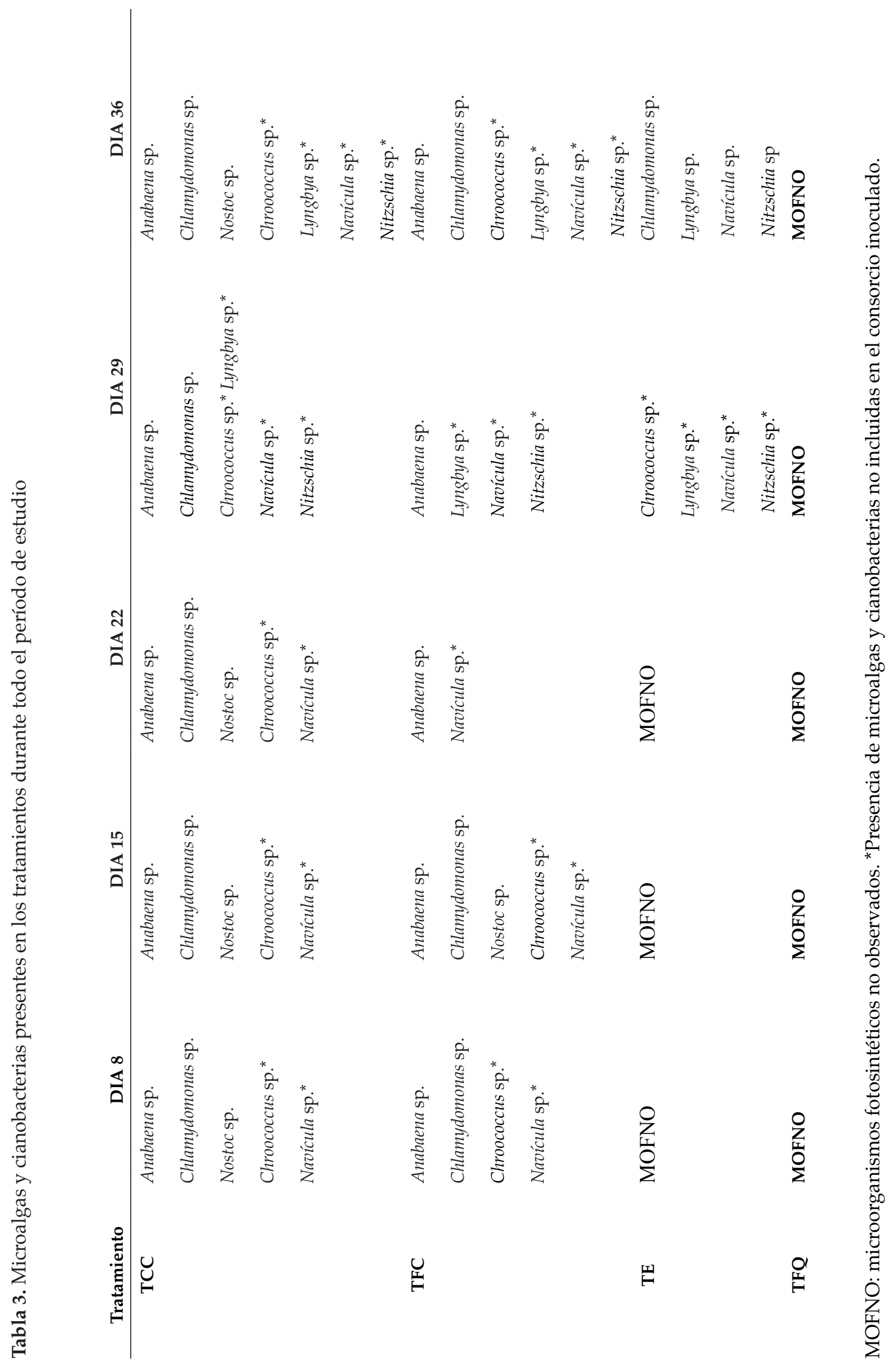




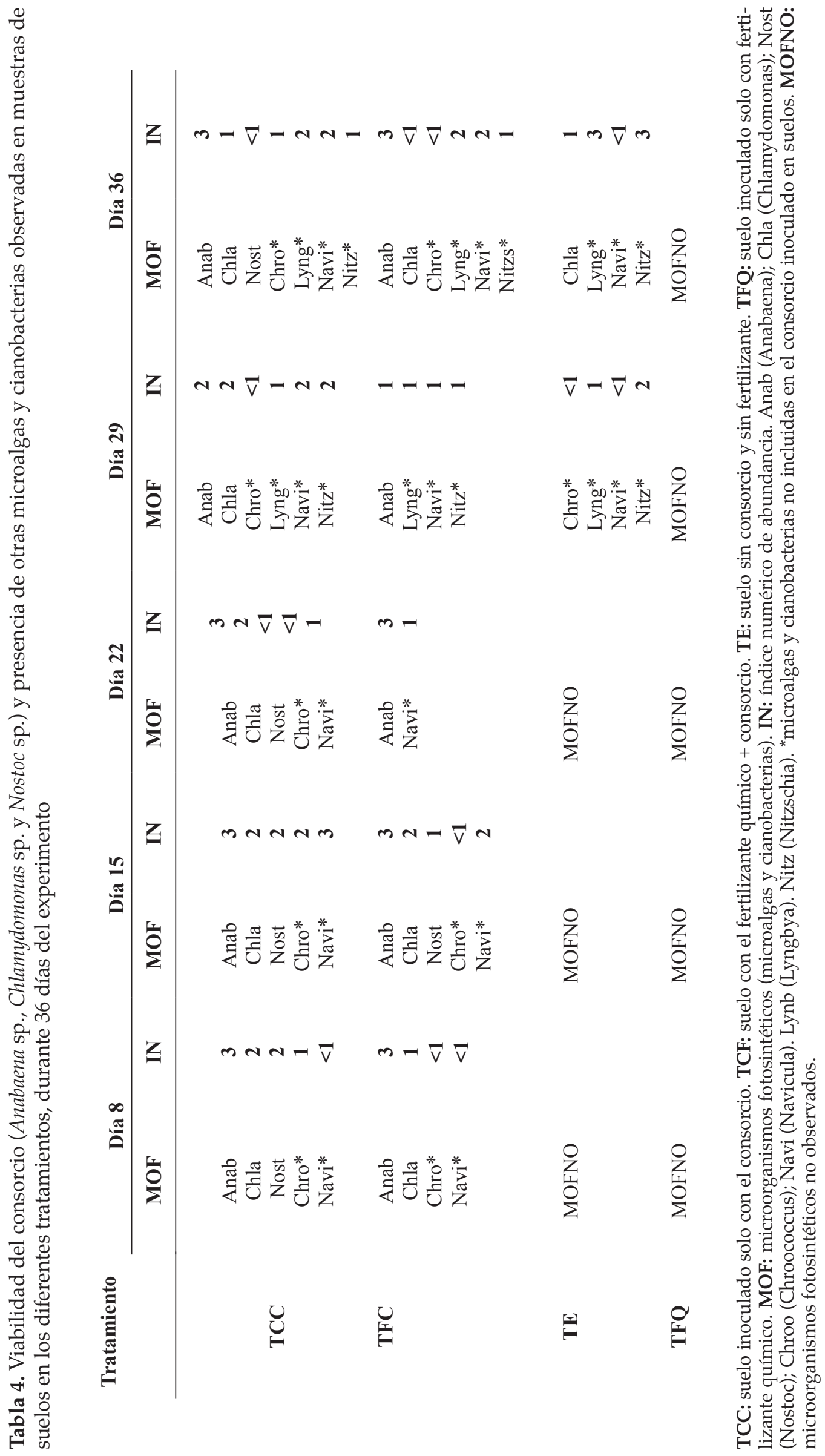


respectivamente. Mientras que, el orden de abundancia de las taxa fue: Barnadesia sp.>Chromolaena sp.>Pennisetum clandestinum $>$ Coriandrum sp.> Trifolium repens $>$ Cichorium intybus $>$ Urtica urens. En cambio en el tratamiento TCF, se registraron 6 taxa y 107 plantas, con el siguiente orden de abundancia: Pennisetum clandestinum $>$ Barnadesia sp.>Chromolaena sp.>Coriandrum sp.>Trifolium repens $>$ Urtica urens. Las plantas observadas en todos los tratamientos correspondieron a Chromolaena sp.,Coriandrum sp. y Pennisetum clandestinum (Tabla 5).

Tabla 5. Población de cada taxa de plantas observadas en los tratamientos

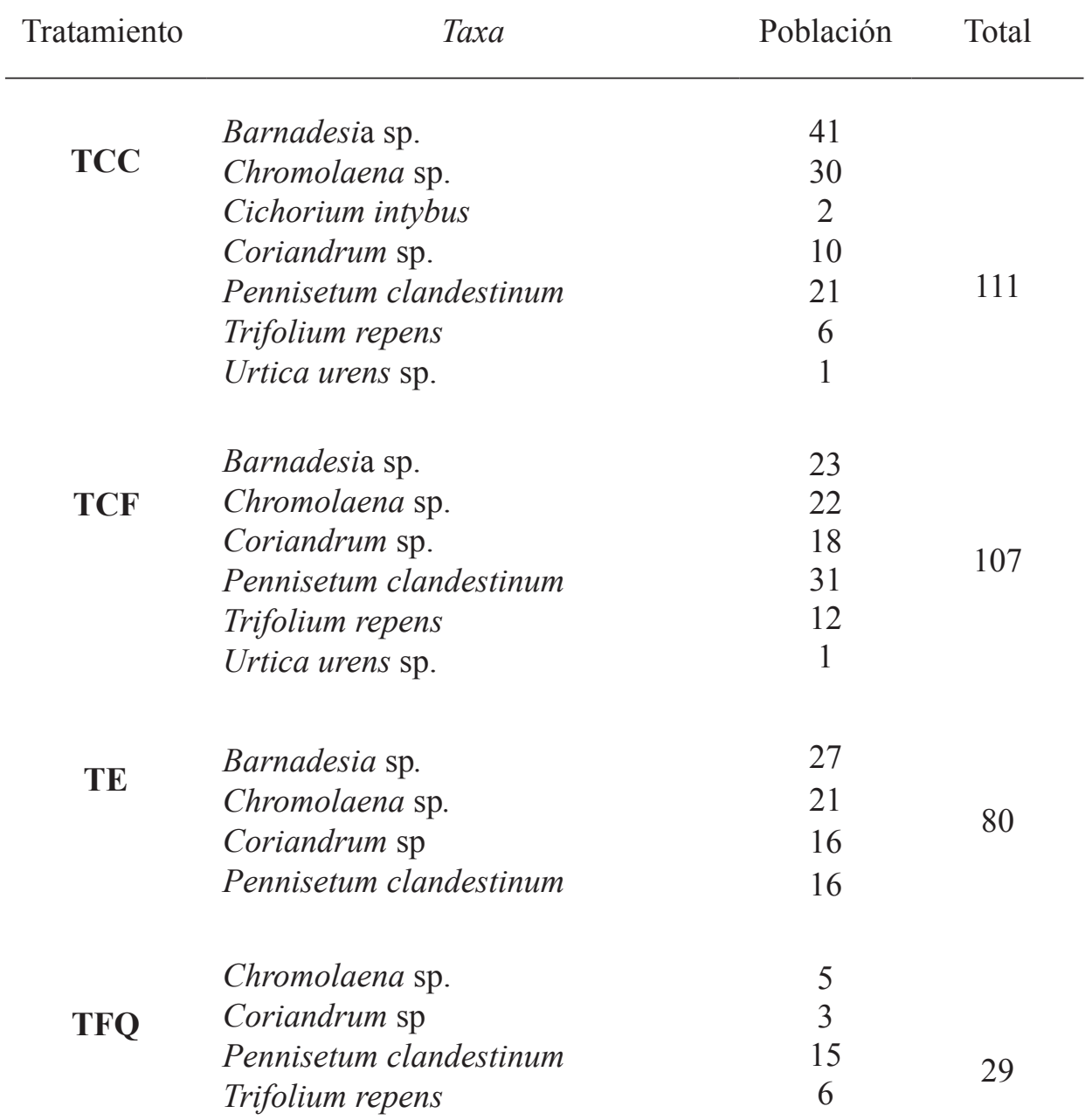

TCC: suelo inoculado solo con el consorcio. TCF: suelo con el fertilizante químico + consorcio. TE: suelo sin consorcio y sin fertilizante. TFQ: suelo inoculado solo con fertilizante químico. 
Por otra parte, se determinó que el número total de plantas por tratamiento estuvo en el siguiente orden: TCC $>$ TCF $>$ TE $>$ TFQ. Así mismo, se evidenció una diferencia significativa $(\mathrm{p}>0.05)$ entre el número de plantas presentes en TCC y $\mathrm{TFQ}$, al realizar una comparación múltiple entre los tratamientos.

\section{Análisis físicoquímico del suelo.-}

En cuanto al $\mathrm{pH}$, los resultados obtenidos indican que los suelos son neutros y como producto de una comparación múltiple, se evidenció que no existió diferencia estadística significativa para las medias del $\mathrm{pH}$ entre los diferentes tratamientos. En cuanto a la materia orgánica, el mayor porcentaje se obtuvo en TCF con 4,50\% ( $p>0.05$ ), seguido del TCC con 2,24\%. En cambio en el tratamiento solo con fertilizante químico se obtuvo el contenido menor con $0,62 \%$.

Los valores más elevados de nitrógeno se presentaron en los tratamientos TCF y TCC, con $0,227 \%$ y $0,110 \%$, pero no se detectaron diferencias significativas. Estos resultados reflejan que tanto el suelo inoculado con el consorcio, como el tratado con la combinación del fertilizante químico y el consorcio, aportan el mayor contenido de nitrógeno al suelo. Sin embargo, entre TCF y TFQ cuyas medias fueron $0,23 \%$ y $0,03 \%$; respectivamente, sí hubo diferencias significativas ( $p>0.05$ ); lo cual indica que el tratamiento TFQ al final del experimento ha reducido su contenido de nitrógeno total, con respecto a TCF y TFQ.

El contenido de fósforo en suelos analizados previo al estudio, fue ligeramente superior al obtenido en TCC y tratamiento testigo (TE), con valores de 49.60, 42.67 y 40.63 ppm; respectivamente. No obstante, no se encontró diferencias significativas entre estos tratamientos. Al comparar el contenido de $\mathrm{Fe}$, se encontraron valores similares en TE, TCC y TCF, con 99.43, 100.97 y 101.90 ppm; respectivamente. En cambio, entre TCF y SAI, se encontró diferencias significativas $(\mathrm{p}>0.05)$.

En cuanto al K, Ca y Mg, los valores ligeramente superiores se observaron también en TCF y TCC, con relación a los demás tratamientos y sin diferencias significativas entre ambos. Solamente en el suelo inoculado con agua destilada, se encontraron valores de estos cationes menores a los tratamientos evaluados (Tabla 6).

Tabla 6. Análisis físicoquímico de suelos ( $\mathrm{pH}, \mathrm{MO}, \mathrm{N}, \mathrm{P}, \mathrm{Fe}, \mathrm{K}, \mathrm{Ca}$ y Mg) sometidos a los diferentes tratamientos con el consorcio y con el fertilizante químico

\begin{tabular}{|c|c|c|c|c|c|c|c|c|}
\hline Tratamiento & pH & $\begin{array}{l}\text { MO } \\
(\%)\end{array}$ & $\begin{array}{c}N \\
(\%)\end{array}$ & $\begin{array}{c}\mathbf{P} \\
(\mathbf{p p m})\end{array}$ & $\begin{array}{c}\mathrm{Fe} \\
(\mathrm{ppm})\end{array}$ & $\underset{(\mathrm{cmol} / \mathrm{Kg})}{\mathrm{K}}$ & $\begin{array}{c}\mathrm{Ca} \\
(\mathrm{cmol} / \mathrm{Kg})\end{array}$ & $\begin{array}{c}\mathrm{Mg} \\
(\mathrm{cmol} / \mathrm{Kg})\end{array}$ \\
\hline SAI & 7.540 & 1.930 & 0.010 & 49.600 & 52.200 & 0.920 & 12.430 & 4.100 \\
\hline TCC & 7.657 & 2.243 & 0.110 & 42.667 & 100.967 & 0.957 & 13.357 & 3.970 \\
\hline TCF & 7.213 & 4.500 & 0.227 & 35.767 & 101.900 & 1.103 & 13.893 & 4.257 \\
\hline TE & 7.460 & 1.563 & 0.080 & 40.633 & 99.433 & 0.767 & 10.527 & 3.397 \\
\hline TFQ & 7.030 & 0.617 & 0.033 & 37.067 & 70.533 & 0.893 & 11.217 & 3.850 \\
\hline
\end{tabular}

SAI: suelo antes del experimento de inoculación. TCC: suelo inoculado solo con el consorcio. TCF: suelo con el fertilizante químico + consorcio. TE: suelo sin consorcio y sin fertilizante. TFQ: suelo inoculado solo con fertilizante químico. 


\section{DISCUSIÓN}

A partir delas muestras de suelos y de rizósfera de las plantas leguminosas: Phaseolus vulgaris (fréjol), Vicia faba (haba), Lupinus mutabilis (chocho) y Pisum sativum (arveja), se identificaron 7 taxa de microalgas (Chlamydomonas sp.,Chlorella sp., Scenedesmus sp., Chlorococcum sp., Desmodesmus sp., Navícula sp. y Pediastrum sp.) y 7 taxa de cianobacterias filamentosas (Nostoc sp., Calothrix sp., Anabaena sp., Phormidium sp., Pseudanabaena sp., Lyngbya sp. y Leptolyngbya sp.); de las cuales 3 presentan heterocistos, como evidencia de ser fijadoras de nitrógeno corresponden a Nostoc sp., Calothrix sp. y Anabaena sp.

En todas las muestras inoculadas con el fertilizante Nitrofoska y el medio de cultivo BG- $11_{0}$ hubo crecimiento de microalgas y cianobacterias; pero con una mayor diversidad de microalgas en muestras de rizósfera de las leguminosas, inoculadas con el fertilizante y con presencia de Chlamydomonas sp., Navícula sp., Scenedesmus sp., Desmodesmus sp., Pediastrum sp. y de las cianobacterias, Anabaena sp., Nostoc sp., Calothrix sp., Lyngbya sp., Pseudanabaena sp. y Leptolyngbya sp. Mientras que, la microalga con la mayor frecuencia de observación en todas muestras correspondió a Chlamydomonas sp., seguida de Lyngbya sp. Aun cuando, el fertilizante Nitrofoska estimuló el crecimiento de microalgas y cianobacterias; el uso del medio BG- $11_{0}$ contribuyó de una manera más eficiente a la proliferación de cianobacterias fijadoras de nitrógeno y de otras sin heterocistos; además de condicionar la presencia de microalgas (Rippka et al., 1979).

La presencia de microalgas y de cianobacterias está demostrada en diferentes tipos de suelos tanto cultivados como no utilizados por el hombre (Metting, 1981). La distribución y la diversidad de microalgas y cianobacterias está influenciada por el contenido de nutrientes, $\mathrm{pH}$, humedad, luz, tipo de manejo del suelo (Alexander, 1982). De tal forma que pueden predominar las microalgas clorofitas, diatomeas o cianobacterias. Es así como, Lin et al. (2013), han descrito características morfológicas y mediante secuenciación del rDNA una amplia diversidad de microalgas y cianobacterias, pertenecientes a los géneros Chlorella, Navicula, Nitzschia, Pinnularia, Microcoleus, Anabaena, Lyngbya, Oscillatoria, Phormidium, Nostoc y Chlamydomonas, entre las 74 taxa reportadas en suelos.

La estructura, ecología y taxonomía de cianobacterias y microalgas edáficas también ha sido descrita por Salaru et al. (2008); la cual está relacionada con la presencia de ecobiormorfos. Tal es el caso de las cianobacterias filamentosas con o sin vaina mucilaginosas ó las cianobacterias fijadoras de nitrógeno (Anabaena, Nostoc, Cylindrospermum), reconocidas por ser indicadoras de la fertilidad natural de suelos y cuya dinámica de población dependerá del uso racional de los suelos para la agricultura o del uso excesivo de fertilizantes para el cultivo masivo de plantas de interés económico.

En el presente estudio, fueron identificadas y aisladas cepas de Anabaena sp., Nostoc sp. y Calothrix sp.; lo que sugiere que estas cianobacterias pueden contribuir con la fertilidad de los suelos cultivados con las leguminosas, como producto de la fijación del nitrógeno atmosférico. Estos resultados también son similares a los reportados por Acosta et al., (1996), quienes identificaron a Anabaena, Nostoc, Oscillatoria, Spirulina, Nodularia y varias especies de diatomeas pennadas en rizósfera de la leguminosa Vigna unguiculata. Tales cianobacterias parecen ser cosmopolitas y frecuentes en diversos tipos de suelos; con lo cual se presenta la factibilidad de aplicar las metodologías para su aislamiento, cultivo y producción de biomasa para fines agrícolas.

En relación las cianobacterias Anabaena sp. y Nostoc sp., procedentes de las muestras de suelos y de rizósfera de las leguminosas evaluadas, y crecidas en cultivos discontinuos alimentados en medio BG-11 $1^{\prime}$ se logró establecer un protocolo para la producción y mantenimiento de consorcios con estas cianobacterias fijadoras de nitrógeno de acuerdo al medio de cultivo y condiciones de escalado de los cultivos discontinuos. La relación proporcional de Anabaena sp., Nostoc sp. y Chlamydomonas sp., de 3:1:3, es indicativo del predominio de Anabaena como cianobacteria fijadora de nitrógeno en el consorcio inoculado y 
de su permanencia en cuanto a viabilidad durante todo el período del estudio cuando fue inoculado dicho consorcio en suelos. El cultivo masivo de consorcios microbianos permite incrementar la eficiencia en cuanto a la producción de biomasa microalgal para biorremediación, biofertilizantes o producción de metabolitos de interés. Tal es el caso, de un consorcio conformado por Nostoc calcicola, Nostoc spongiaeformae, Nostoc linckia y Nostoc muscorum, aislado de suelos y cultivado en medio BG-11 $1^{\prime}$ en una proporción de 1:1:1:1 para la producción de exopolisacáridos, reducción de la salinidad en suelos y mejoramiento en la germinación de semillas de maíz, trigo y arroz (Arora et al., 2010).

El uso de cianobacterias como acondicionadoras de suelos mediante consorcios, también ha sido descrita por Amal et al. (2010), quienes a partir de cianobacterias fijadoras de nitrógeno (Nostoc muscorum, Nostoc humifusum, Anabaena oryzae y Wollea sp.) y de no fijadoras (Phormidium sp. y Spirulina platensis), cultivadas por separado en medio BG11 y Spirulina platensis, en medio Zarrouk fueron combinadas posteriormente en proporciones iguales para conformar dicho consorcio. De tal manera que, lograron demostrar la estabilidad del consorcio con cianobacterias fijadoras de nitrógeno en cultivos discontinuos alimentados con medio BG-11 0 durante 3 meses consecutivos, para ser inoculados luego a suelos.

La viabilidad del consorcio inoculado en suelo (TCC), en función de la presencia de Anabaena sp., Nostoc sp. y Chlamydomonas sp., quedó demostrada (Tabla 3), en virtud de que estos microorganismos fotosintéticos se mantuvieron durante los 36 días del estudio. En cambio, el suelo inoculado con el fertilizante y el consorcio (TFC), Anabaena sp. se mantuvo durante todo el experimento. Sin embargo, Nostoc sp. aparentemente fue observado hasta el día 15. Mientras que, en el tratamiento no inoculado (TE) no observada Anabaena sp., ni Nostoc sp., inoculadas solo en los tratamientos TCC y TFC. Esto demuestra una alta viabilidad de las cianobacterias inoculadas e incluso de Chlamydomonas sp.; a pesar de que el volumen del consorcio fue diluido al 40\% y al $80 \%$ (v/v) en el tratamiento TCC y TCCF, respectivamente.
Así mismo, se destaca una diversidad de cianobacterias y de microalgas al final del estudio, con presencia de Anabaena sp., Nostoc sp., Lyngbya sp., Chroococcus sp., Chlamydomonas sp., Navícula sp. y Nitzschia sp. en el tratamiento TCC y con la presencia de estos mismos microorganismos en TCF, a excepción de la cianobacteria Chroococcus sp. Lo que permite inferir que tanto en el tratamiento TCC y TFC, inoculados con el consorcio se identificaron las mismas taxa y con la presencia del consorcio al menos sus tres integrantes en TCC. De tal manera que, estos resultados sugieren una participación significativa del consorcio con su aporte de biomasa y de nutrientes al suelo.

La diversidad de otros grupos de microalgas o de cianobacterias puede observarse en suelos inoculados con cianobacterias. En un trabajo realizado con Nostoc sp. como biofertilizante en suelos para el cultivo de girasol (Helianthus annuus L.), se aplicaron varios tratamientos: Testigo; Inoculado (6 $\mathrm{g} \mathrm{ha}^{-1}$ de biomasa pura); Fertilizado (fosfato-diamónico $70 \mathrm{~kg} \mathrm{ha}^{-1}$ ); Fertilizado e Inoculado. Entre los resultados obtenidos, se observó que en todas las muestras predominaron las diatomeas pennadas (Bacillariophyta), siendo el suelo inoculado, el único que presentó Nostoc sp. y otras cianobacterias como grupo subdominante. En este caso, Lyngbya fue el género más abundante. Mientras que, en las muestras Testigo, únicamente se observaron diatomeas pennadas y no se identificaron cianobacterias, ni Chlorophytas (Sotelo et al., 2008). Estos resultados, coinciden con los obtenidos en el presente trabajo debido a que además, de las cianobacterias inoculadas, pueden identificarse otros grupos de microalgas, tomando en cuenta que en muchos casos, en los suelos no inoculados, no se evidencia la presencia del consorcio o de cianobacterias que previamente han sido inoculadas.

En cuanto a la viabilidad de cianobacterias aisladas de rizósfera de plantas de arroz, se ha reportado un estudio en el que también se ha evaluado su persistencia en el suelo, en las raíces, y su capacidad para promover el crecimiento de plantas y mejorar la fertilidad del suelo en condiciones de invernadero. En dicho 
estudio, de 20 cepas inoculadas, 15 de estas se mantuvieron viables en el suelo superficial hasta la cosecha del cultivo. Mientras que, 9 mostraron un crecimiento visible en la rizósfera. Sin embargo, sólo 12 cepas persistieron en las raíces, y las otras muestras fueron dominados por las cepas nativas Nostoc y cianobacterias del grupo LPP (Prasanna et al., 2009).

En otro estudio realizado para demostrar la persistencia de una cepa de Nostoc y su contribución a la fertilidad del suelo y al crecimiento de arroz, se encontró que después de tres semanas de la inoculación sobre $90 \%$ de la totalidad, se observaron las superficies de los suelos inoculados uniformemente cubierta por biopelículas de Nostoc en combinación con otras cianobacterias. Por lo que, estas observaciones sugirieron que la cepa de Nostoc inoculada pudo estabilizarse y crecer en las superficies de los suelos experimentales (Maqubela et al., 2009). Al respecto, la biomasa microalgal es fundamental para el mantenimiento de las funciones del suelo; ya que representa la principal fuente de las enzimas que regulan los procesos de transformación de elementos en los suelos. Además de, que también controla la acumulación y descomposición de la materia orgánica, y sirve como indicador precoz de los cambios en la gestión del suelo, de la contaminación por metales pesados y de las prácticas de fertilización (Prasanna et al., 2009).

La elevada diversidad (6 taxa) y población de plántulas crecidas en los tratamientos TCC (111) y TCF (107), con respecto al testigo (80) y TFQ (29), sugiere que la introducción del consorcio puede favorecer las condiciones estructurales y funcionales del suelo, para que sea estimulada la germinación de semillas de plantas silvestres. En este sentido se prevé que el consorcio inoculado, contribuya a incrementar la materia orgánica y de nutrientes para favorecer el crecimiento de estas plántulas. Tal es el caso, de un estudio realizado con la inoculación de Nostoc commune en un campo de pastoreo de vacunos, en el cual se evaluó la posible relación positiva entre la presencia de esta cianobacteria y la germinación de semillas y la emergencia de plántulas. Al respecto, se observó que los micrositios cubiertos por la cianobacteria mostraron un mayor número de plántulas en otoño, invierno y primavera, y que la mayor abundancia de plántulas (68,3 plántulas/ $\mathrm{dm}^{2}$ ) ocurrió en las microdepresiones con Nostoc. En tal sentido, los autores concluyeron que la presencia de las biodermas de la cianobacteria tiene un significativo impacto ecológico, al mejorar las propiedades del suelo, estimulando la germinación, la emergencia y el establecimiento de las plantas anuales; tales como Hordeum pusillum, que bajo la influencia de la cianobacteria, el suelo fue menos alcalino y se incrementó su contenido de materia orgánica, carbono y nitrógeno (Ansín et al., 2002).

La baja densidad de población de estas plántulas crecidas en el tratamiento solo con el fertilizante comercial (TFQ), posiblemente sea debido al exceso de algún compuesto inhibitorio. No obstante, población similar en cuanto al número de plantas y similar diversidad de plantas obtenida en este tratamiento como en TCC (Tabla 5); pudiera deberse a que la inoculación del consorcio, posiblemente neutralice algún factor inhibitorio o mejore la disponibilidad de nutrientes como para que se exhiba una elevada eficiencia en cuanto a la fertilidad del suelo para el crecimiento y diversidad de las plantas; tal como se obtuvo en el tratamiento con solo la inoculación del consorcio.

De acuerdo con el análisis físico-químico realizado al suelo antes y después de ser inoculado con el consorcio y con el fertilizante químico, se observó que no hubo variabilidad del $\mathrm{pH}$ entre los tratamientos aplicados. No obstante, estudios realizados por Amal et al. (2010), en cuanto a inoculación de cianobacterias en suelos, encontraron que el $\mathrm{pH}$ y la conductividad presentaron un ligero decrecimiento, debido a que el uso de biofertilizantes nitrogenados con las cianobacterias podría causar una cierta acidificación de los suelos. Esto sugiere que, la aplicación de consorcios con cianobacterias a suelos alcalinos puede resultar en una agregación de estos y a la vez una disminución del pH (Sutton et al., 1991).

En cuanto al porcentaje de materia orgánica se obtuvo un incremento de 3.63 veces en el suelo 
inoculado con el consorcio, en relación al suelo solo con el fertilizante químico. En cambio, en el suelo inoculado con el consorcio y el fertilizante (TFC) con respecto al tratamiento con el consorcio (TCC) el aumento de la materia orgánica fue de 2.01 veces. Estos resultados coinciden con los reportados por (Prasanna et al., 2009), quienes luego de inocular 20 cepas de cianobacterias aisladas de rizósferas de diversas variedades de arroz y de trigo, tales como de Anabaena, Calothrix, Nostoc y Hapalosiphon, detectaron un aumento de la relación Carbono microbiano (Cmic) con respecto al Carbono orgánico (Corg); obteniéndose un rango entre 0,15 a 6,17; lo cual significó una elevada relación Cmic / Corg, como producto de la inoculación de las cianobacterias fijadoras de nitrógeno.

En suelos salinos de Bangladesh e inoculados con 8 cepas de cianobacterias, previamente aisladas, identificadas y mantenidas en cultivos monoespecíficos, también se determinó un incremento en la materia orgánica, N y P del suelo, con relación a los suelos no tratados con consorcios. De tal manera, que estos pueden ser utilizados con eficacia para mejorar la fertilidad del suelo de suelo salino (Aziz y Hashem, 2003).

Las evaluaciones del nitrógeno presente en el suelo sometido a los diferentes tratamientos, evidencian que efectivamente la combinación del fertilizante químico con el consorcio de cianobacterias (TFC) aporta el mayor contenido de nitrógeno al suelo. Sin embargo, si se compara el contenido de nitrógeno entre el tratamiento TCC y el TFQ, se refleja un incremento de 3,33 veces cuando el suelo es inoculado con el consorcio.

El incremento del nitrógeno en suelos tratados con cianobacterias también ha sido reportado por otros autores. Entre los cuales se describe el trabajo de Maqubela (2009), quien detectó un aumento del $\mathrm{N}$ del suelo en un $40 \%$ y un $17 \%$ en dos tipos de suelos, Guquka y Hertzog, respectivamente, cultivados con maíz. Además, determinaron un aumento de materia seca del maíz en un 40 y $49 \%$, cultivado en estos suelos e inoculado con la cepa Nostoc 9v. En un estudio realizado por Amal et al. (2010), adicionaron cepas de Nostoc, Anabaena oryzae, Wollea sp., Phormidium sp.y de Spirulina sp. en suelos cultivados con Phaseolus vulgaris y reportaron una mejora de la disponibilidad de $\mathrm{N}$, P y K de manera significativa; lo que les permitió proponer que, la $1 / 4 / 4$ parte $\mathrm{o} \frac{1}{1} / 2$ del nitrógeno requerido en los fertilizantes comerciales, puede ser suministrado por las cianobacterias inoculadas.

El contenido de fósforo en cada uno de los tratamientos, no presentó diferencia significativa; lo cual sugiere que al menos no hubo una variabilidad evidente en cuanto al enriquecimiento con el fósforo. Sin embargo, el suelo presentó un contenido de 42.67ppm con el consorcio. Mientras que, con el fertilizante fue de 37.07ppm. Al respecto, en suelos tratados con cianobacterias y cultivados con arroz en Bangladesh, tampoco detectaron variabilidad en el contenido de P del suelo después de la cosecha; aunque el mayor contenido de P de 19.1 ppm se registró en el tratamiento (RFD-40\% N + cianobacterias); el cual correspondió a la dosis del fertilizante recomendada (RFD), con un 40\% de nitrógeno y con la mezcla de cianobacterias. Mientras que el menor contenido de $\mathrm{P}$, se observó en tratamiento control (Aziz y Hashem, 2003).

Con respecto al contenido de azufre en los suelos tratados con la combinación: consorcio + fertilizante (TCF), o en el testigo, o antes del experimento, se obtuvo el mayor contenido de azufre en TCF; lo cual sugiere que el fertilizante comercial aplicado es rico en este elemento y a pesar de que en el tratamiento TCC se obtuvo un contenido de 73.17ppm, este contribuyó a incrementar el nivel de azufre en TCF con 109.79 ppm. Los resultados reportados por (Aziz y Hashem, 2003) indicaron para el azufre que su disponibilidad no fue significativamente afectada por la aplicación de las cianobacterias. Sin embargo, las parcelas tratadas con estas, presentaron un contenido de azufre más alto, que aquellos que no fueron inoculados; de tal manera que se produjo también para el tratamiento (RFD20\% N + cianobacterias) el mayor contenido de azufre con 15.8 ppm y el más bajo en el control con 14.0 ppm.

Entre los cationes que presentaron cierta variabilidad en los suelos inoculados con el consorcio 
(TCC), los tratados con el fertilizante y el consorcio (TCF) y los tratados con el fertilizante químico (TFQ), se distinguieron el Fe y $\mathrm{Mn}$; puesto que el $\mathrm{B}, \mathrm{K}, \mathrm{Ca}, \mathrm{Mg}, \mathrm{Cu}$ y $\mathrm{Zn}$ no presentaron diferencias significativas entre estos tratamientos. El Fe incrementó en el suelo TCC y TCF en 1,43 y 1,44 veces, respectivamente con respecto al fertilizado solamente con el producto químico (TFQ), En cambio, el Mn, registró un aumento de 1.32 en relación al tratado con el fertilizante químico. Respecto a estos resultados se ha descrito por Al-Khiat (2006) un incremento de $\mathrm{Fe}, \mathrm{Mn}$ y $\mathrm{Zn}$ en aquellos suelos inoculados con Anabaena oryzae y Nostoc ellipsoidum, en comparación al suelo no inoculado y antes de un transplante de las plántulas de Lycopersicon esculentum. En relación a estos resultados se infiere que tanto el contenido Fe como el Mn, pueden ser modulados por el aporte del consorcio y del fertilizante químico adicionado al suelo.

\section{CONCLUSIONES}

- Los resultados obtenidos en el presente estudio permiten sugerir que el uso del consorcio con Anabaena, Nostoc y Chlamydomonas constituye una alternativa para mejorar la disponibilidad de nutrientes, materia orgánica y factibilidad de germinación de semillas.

- La combinación de dicho consorcio con el fertilizante químico en cuanto a incremento de la fertilidad del suelo, sugiere una opción eficiente ante el uso directo de los fertilizantes.

- Se establece un protocolo para la producción y mantenimiento de consorcios con cianobacterias fijadoras de nitrógeno de acuerdo al tipo de suelo, medio de cultivo y condiciones de escalado de los cultivos discontinuos.

- La conformación de consorcios cianobacterias-microalgas en biotecnología agrícola permite ampliar el espectro de microorganismos utilizados como acondicionadores de suelos.

\section{AGRADECIMIENTOS}

A la Escuela de Bioanálisis de la Pontificia Universidad Católica del Ecuador y al Centro de Biología de la Universidad Central del Ecuador, por haber facilitado las condiciones para la realización de la presente investigación. Este trabajo ha sido financiado por el Programa Becas Prometeo de la Secretaría Nacional de Educación Superior, Ciencias y Tecnología de la República del Ecuador.

\section{REFERENCIAS BIBLIOGRÁFICAS}

Acosta K, Santos R, Silva S y Esparza D. 1996. Evaluación cualitativa y cuantitativa de microalgas en la rizosfera del cultivo de frijol. Revista de la Facultad de Agronomía, 13 (1): 13-24.

AGROCALIDAD. 2011. Manual de requisitos para los usuarios de Agrocalidad. Agencia Ecuatoriana de Aseguramiento de la Calidad del Agro. Dirección Administrativa, Financiera y Tecnológica. 165 pp.

Aguirre J, Irizar M, Durán A, Grajeda O, Peña M, Loredo C y Guitierrez M. 2009. Los Biofertilizantes microbianos: alternativa para la agricultura en México. Centro de Investigación Regional Pacífico Sur. Campo Experimental Rosario Izapa.

Alexander M. 1982. Most probable number methods for microbial populations. American Society of Agronomy Inc., 815-820.

Al-Khiat A. 2006. Effect of Cyanobacteria as a Soil Conditioner and Biofertilizer on Growth and Some Biochemical Characteristics of Tomato (Lycopersicon esculentum L).Thesis Degree of Master of Science in Microbiology (Algae).King Saud University. Arabia Saudita.

Amal Z, Hegazi A, Mostafa, Hamdino M y Ahmed I. 2010. Influence of different cyanobacterial application methods on growth and seed production of common 
bean under various levels of mineral nitrogen fertilization. Nature and Science, 8 (11): 183-193.

Ansín OE, Deregicus A y Landfranco JW. 2002. Papel del alga Nostoc commune y efecto del pastoreo por vacunos sobre la colonización de suelos alcalinos en la Pampa deprimida. Ecología Austral, 12: 135-142.

Arango Pulgarín G y Pérez Naranjo J. 2005. Determinación de nitratos y amonio en muestras de suelo mediante el uso de electrodos selectivos. Revista de la Facultad Nacional de Agronomía. Universidad Nacional de Colombia, 58 (1), 2733-2740.

Armenta A, García C, Camacho R, Apodaca M y Montoya L. 2010. Biofertilizantes en el desarrollo agrícola de México. Ra Ximhai Revista de sociedad, cultura y desarrollo sustentable, 51-54.

Arora M, Kaushik A, Rani N y Kaushik C. 2010. Effect of cyanobacterial exopolysaccharides on salt stress alleviation and seed germination. Journal of Environmental Biology, 31(5): 701-704.

Aziz M y Hashem M. 2003. Role of Cyanobacteria in Improving Fertility of Saline Soil. Pakistan Journal of Biological Sciences, 6 (20):1751-1752.

Báscones Merino E. 2004. Análisis de Suelos y Consejos de Abonado. Escuela Universitaria de Ingeniería Agrícola. Diputación Provincial de Valladolid, España. Primera Edición. 65 pp.

Benitez Burraco SA. 2005. Avances recientes en Biotecnología vegetal e ingenieria de plantas. Primera Edición. Editorial Reverté. 232 pp.

Bennet A y Bogorad L. 1973. Complementary chromatic adaptation in a filamentous blue- green alga. Journal of Cell Biology, 58: 419-435.
Eaton A, Clesceri L, Greenberg A y Franson M. 1995. Key for identification of freshwater algae commun in water supplies and polluted water. En Standard Methods for the Examination of water and wasterwater. American Public Health Association. Washington.

Komárek J y Anagnostidis K. 2005. Teil: Cyanoprokaryota. Oscillatoriales. Subwasserflora von Mitteleuropa. Elsevier $\mathrm{GmbH}$, München, Alemania.

Lin CS, Chou TL y Wu JT. 2013. Biodiversity of soil algae in the farmlands of mid-Taiwan. Botanical Studies, 54: 41-53.

Maqubela M. 2009. Bioconditioning and nitrogen fertility effects of selected cyanobacteria strains on two degraded soils in the Eastern Cape. Sudáfrica. Thesis of Doctor of Philosophy in Soil Science. Faculty of Science \& Agriculture. University of Fort Hare. Sudáfrica.

Maqubela M, Mnkeni P, Issa O, Pardo M y D’Acqui L. 2009. Nostoc cyanobacterial inoculation in South African agricultural soils enhances soil structure, fertility, and maize growth. Plant y Soil, 315(12): 79-92.

Masabni JG y Lillard P. 2011. Jardinería Fácil Fertilización. AgriLIFE EXTENSION, 6pp. www.tamuc.edu/academics/colleges.

Marker A, Nusch E, Rai H y Riemann B. 1980. The measurement of photosynthetic pigments in freshwater and standarization of methods: conclusions and recommendations. Archives of Hydrobiology Egebn. Limnology, 14: 91-106

Metting B. 1981. The systematics and ecology of soil algae. The Botanical Review 47 (2): 196-273.

Monteros S y Iglesias R. 2005. Inoculación de cianobacterias en el cultivo de soja, efectos sobre la infectividad de Bradyrhizobium 
japonicum y la producción de materia seca. Argentina: Comunicaciones científicas y tecnológicas.

Morales E, Luna V, Navarro L, Santana V, Gordillo A y Arévalo A. 2013. Diversidad de microalgas y cianobacterias en muestras provenientes de diferentes provincias del Ecuador destinadas a una colección de cultivos. Revista Ecuatoriana de Medicina y Ciencias Biológicas (XXXIV), (1-2) : 129-149.

Prasanna R, Nain L, Ancha R, Jadhay S, Joshi M y Kaushik B. 2009. Rhizosphere dynamics of inoculated cyanobacteria and their growth-promoting role in rice crop. Egyptian Journal of Biology, (11): 26-36.

Red BIOFAG. 2007. Red Iberoamericana de Fertilizantes Biológicos de la Agricultura y el Medio Ambiente. Retrieved from www. biofag.org.ar

Rippka R, Deruelles J, Waterbury J, Herdman M y Stainer R. 1979. Generic assigments, strain histories and properties of pure cultures of cyanobacteria. Journal General of Microbiology, 111: 1-61.

Rumrich U, Lange-Bertalot H, y Rumrich M. 2000. Diatoms of the Andes from Venezuela to Patagonia/Tierra del Fuego and two additional contributions. Germany. 672 pp.

Salaru V, Trofim A, Melniciuc C, y Donu N. 2008. Taxonomic and ecologic structure of communities of edaphic algae from the agrophytocenoses of the northern districts of Moldova. Plant Development, 15: 3-6.
Sotelo C, Iglesias M, y Punos L. 2008. Efecto de la coinoculación cianobacterias - Bradryrhizobium japonicum sobre la actividad biológica del suelo. Comunicaciones cientificas y tecnológicas. Universidad Nacional del Nordeste, Argentina.www.unne.edu.ar/ unnevieja/investigacion/com2008.

Strickland J. y Parsons T. 1972. A practical handbook of seawater analysis. $2^{\text {nd }}$ Edition. Fisheries Research Board of Canada Bulletin, Ottawa. 327 pp.

Suquilanda M. 2008. El deterioro de los suelos en Ecuador y la producción Agrícola. XI Congreso Ecuatoriano de la Ciencia del Suelo (pp. 1-56). Quito: Universidad Central del Ecuador.

Sutton S, Barret G, yTaylor D. 1991. Microbial metabolic activities in soils of old-field communities following eleven years of nutrient enrichment. Environmental Pollution, 73: 1-10.

Terry A, Leyva A, y Hernández A. 2005. Microorganismos benéficos como biofertilizantes eficientes para el cultivo de tomate. Revista Colombiana de Biotecnología, VII (2): 47-54. 